\title{
Immediate serial recall of mixed letter strings'
}

\author{
M. S. MAYZNER AND M. E. TRESSELT
}

NEW YORK UNIVERSITY

This study examined the immediate serial recall of letter strings composed of a mix of high and low frequency consonants, for high and low digram storage Ss. The results clearly showed that high digram storage $S$ s show superior recall to low digram storage Ss for mixed letter strings and that systematic proactive and retroactive effects on recall performance occur with such mixed letter strings.

In a recent study (Mayzner \& Tresselt, 1967) high and low digram storage Ss, as assessed by means of a skeleton-word digram ranking test (Mayzner \& Tresselt, 1962; Tresselt \& Mayzner, 1966), were presented with letter strings that varied in their single-letter and digram frequencies, with the results showing superior recall for the high digram storage Ss for all types of letter strings examined. In the present study high and low digram storage Ss again are presented with letter strings and asked for their immediate serial recall. However, in contrast with previous studies in which the letter strings were composed entirely of elther high frequency or low frequency consonants (Mayzner \& Schoenberg, 1964; Mayzner \& Tresselt, 1967), the present study employs mixed strings, in which the same letter string contains both high and low frequency consonants. Also, the serial position of these high and low frequency consonants are varied systematically in order to provide a preliminary assessment of proactive and retroactive influences of high and low frequency elements within the same letter strings.

Method

One hundred students in introductory psychology classes at NYU were administered the skeletonword digram ranking test (Tresselt \& Mayzner, 1966) and 50 Ss, a high digram storage group (HS) with scores ranging from 17 to $27(\mathrm{~N}=25)$ and a low digram stores group (LS) with scores ranging from 7 to $17(N=25)$. were selected for the presentation of 16 letter strings. These strings were composed of a mixture of high frequency (C, D, F, H, $L, N, R, S$, and $T$ ) and low frequency ( $B, G, J, K, P, Q, V, X$, and $Z$ ) consonants, as given in the single-letter frequency counts of Underwood \& Schulz (1960). Sixteen strings were selected to yield two examples for elght different types of mixed string variations. Eight strings were composed of a $\mathrm{mix}$ of three high frequency letters and six low frequency letters (3H6L) and eight strings were composed of a $\mathrm{mix}$ of three low frequency letters and six high frequency letters (3L6H). The three high or low frequency letters always occurred immediately next to one another, but as a group occupied serial positions $1-3,3-5,5-7$, or $7-9$. The
16 letter strings examined were as follows (with the three high or low frequency letters underlined here to show the reader their serial position in the strings): 3H6L-(1) DTFQBZVJX, (2) SRHVXPZQJ, (3) XQLCNPZVK, (4) BZTNRQKGJ, (5) BKGVFDHZQ, (6) GZXQLCSJP, (7) VPJQXZTDF, (8) ZKQBGJHLR; 3L6H-(1) BKGDTFSRH, (2) ZJQLRHDTC, (3) TNPXVRFDH, (4) DFZJQCSNR, (5) CNFSVBGDH, (6) SDCNPKXHL, (7) LCFHDTXQB, (8) TFDNRHKZV. Since previous studies (Mayzner \& Schoenberg, 1964; Mayzner \& Tresselt, 1967) had shown sequential digram frequencies influenced recall performance, the 16 letter strings were ordered so as to yield sequential digram frequency values of zero or very close to zero.

Each of the 16 letter strings, each nine letters in length and each typed on a $3 \times 5$ in. card in a single horizontal line, were displayed to $S$ for 6 sec. and $S$ was then given $10 \mathrm{sec}$. to write the letters down. Each $S$ received the 16 strings in a different random order and for each recall, $S$ was given a blank card with nine dashes on it and was told to write down the letters he could recall in their correct position and sequence. Results and Discussion

Since the 16 letter strings were composed of a $\mathrm{mix}$ of high and low frequency consonants (1.e., 3H6L or $3 \mathrm{~L} 6 \mathrm{H})$ and the $3 \mathrm{H}$ and $3 \mathrm{~L}$ groupings occupied serial positions 1-3, 3-5, 5-7, and 7-9, percent correct recall was calculated separately for all of these combinations, also taking into account the high and low digram storage conditions (i.e., HS and LS). The results of this analysis are presented in Figs. 1 and 2. Thus, Fig. 1 presents percent correct recall for $3 \mathrm{H}$ mixed with $6 \mathrm{~L}$ and $3 \mathrm{~L}$ mixed with $6 \mathrm{H}$ for the HS and LS groups and for serial positions 1-3, 3-5, 5-7, and 7-9 (i.e., groups HS-3H6L, LS-3H6L, HS-3L6H, and LS-3L6H). Since it was desired also to examine the proactive and retroactive effects involved in the mix of high and low frequency consonants, and since in a previous study (Mayzner \& Tresselt, 1967) HS and LS groups had recelved unmixed strings of nine high frequency or nine low frequency consonants, equivalent percent correct cecall scores were calculated treating the $9 \mathrm{H}$ string as if it were a $3 \mathrm{H} 6 \mathrm{H}$ string and the $9 \mathrm{~L}$ string as if it were a $3 \mathrm{~L} 6 \mathrm{~L}$ string, taking into account equivalent serial position variations. The results of this analysis are presented also in Fig. 1 (i.e., HS-3H6H, LS-3H6H, HS-3L6L, and LS-3L6L). The results show clearly for all HS-LS comparisons that percent correct recall is greater for the HS groups than for the LS groups for both the mixed and unmixed strings and for all serial position variations, thus supporting our previous find- 
ings with the storage variable (Mayzner \& Tresselt, 1967). For example, for the $3 H$ conditions HS recall is superior to LS recall for all eight possible comparisons ( $p<.004$ ) as evaluated with the Sign Test) and identical results occur with the $3 \mathrm{~L}$ conditions. In contrasting $3 \mathrm{H}$ mixed with $6 \mathrm{H}$ versus $3 \mathrm{H}$ mixed with 6L for the HS and LS groups, we find superior recall for the $3 \mathrm{H} 6 \mathrm{H}$ combination over the $3 \mathrm{H} 6 \mathrm{~L}$ combination in seven out of the eight possible comparisons ( $p<.035)$, while in contrasting $3 \mathrm{~L}$ mixed with $6 \mathrm{~L}$ versus 3L mixed with $6 \mathrm{H}$ for the HS and LS groups, we find superior recall for the $3 \mathrm{~L} 6 \mathrm{H}$ combination over the 3L6L combination in seven out of the elght possible comparisons $(p<.035)$.

Figure 2 presents similar analyses as Fig. 1, but for percent correct recall for the $6 \mathrm{H}$ and $6 \mathrm{~L}$ components. Again the HS groups show superior recall to the LS groups on all equivalent comparisons and again the mixed strings analysis reveals equivalent trends. Also, both Figs, 1 and 2 clearly show for all conditions that percent correct recall decreases as serial position values move from 1-3 to 3-5 to 5-7 to 7-9. Overall the results suggest that the digram storage variable continues to play as an important a role with mixed

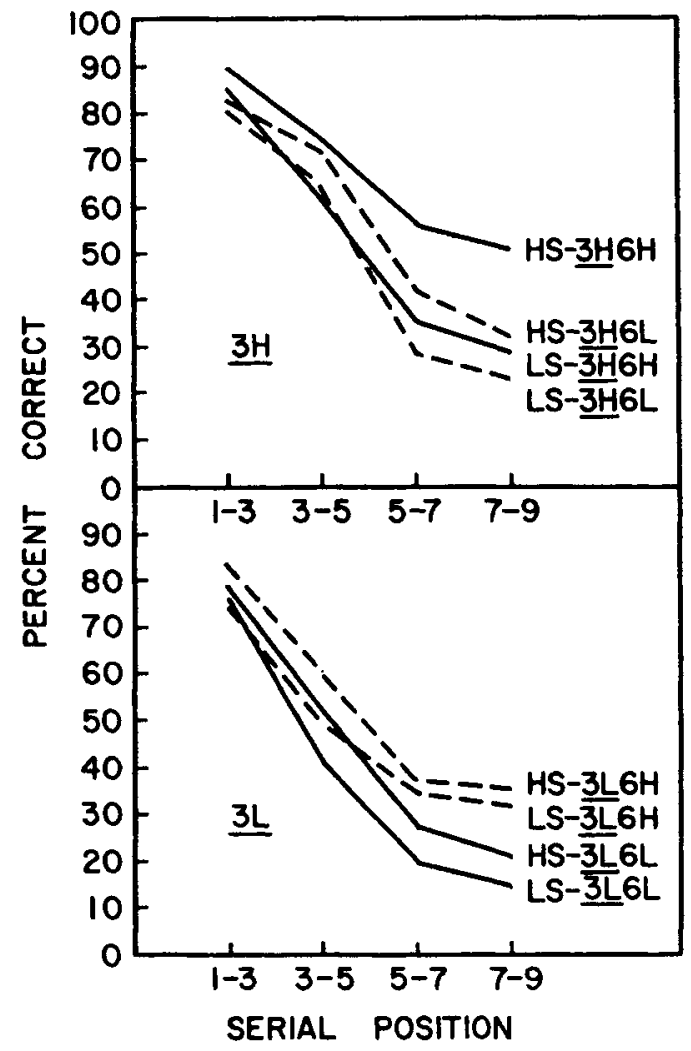

Fig. 1. \% correct recall for HS and LS groups for the $3 H$ and $3 L$ strings as a tunction of serial position.

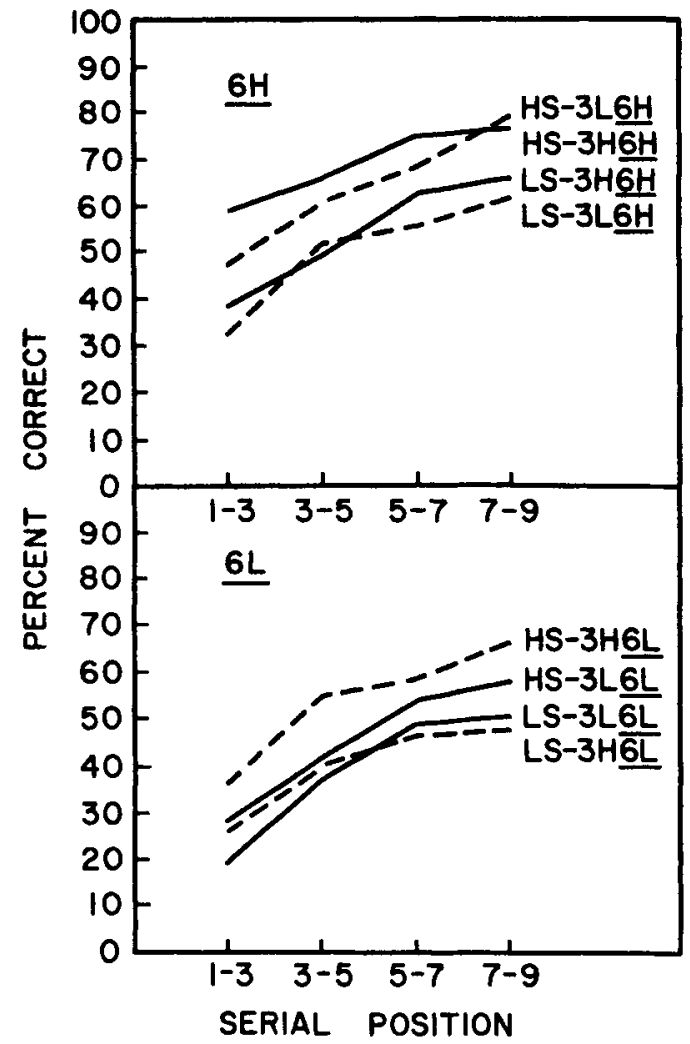

Fig. 2. \% correct recall for the $H S$ and $L S$ groups for $6 H$ and $6 L$ strings as a function of serial position.

strings as it does with unmixed strings and that the percent correct recall of various letter subsets within the total letter string (1.e., $3 \mathrm{H}, 3 \mathrm{~L}, 6 \mathrm{H}$, and $6 \mathrm{~L}$ ) show differential proactive and retroactive variations, which warrants further systematic study.

\section{References}

Mayzner, M. S., \& Schoenberg, K. M. Single-letter and digram frequency effects in immediate serial recall. J. verbal Learn. verbal Behav., 1962, 1, 203-207.

Mayzner, M. S., \& Tresselt, M. E. The ranking of letter pairs and single letters to match digram and single-letter frequency counts. J. rerbal Learn. rerbal Bchav., 1962, 1, 203-207.

Mayzner, M. S., \& Tresselt, M. E. Individual differences in stored digram frequencies and the immediate serial recall of letter and number strings. Psychon. Sci., 1967, 7, 359-360.

Tresselt, M. E., \& Maymer, M. S. Normative solution times for a sample of 134 solution words and 378 associated anagrams. Psychon. Monoar. Suppl., 1966, 1, 293-298.

Underwood, B. J., \& Schulz, R. W. Mcaninglulness and verbal l'arning. Philadelphia: Lippincott, 1960.

\section{Note}

1. This research was supported in part by Cuntract Nonr 285(56) between the Engineering Psychology Branch of the Office of Naval Research and New York University. 